DOI: $10.17234 / S R A Z .65 .49$

\title{
La grammaticografia italiana degli ultimi decenni - nel solco della tradizione classica o al bivio tra l'innovazione e la decadenza?
}

Saša Bjelobaba

Facoltà di Scienze Politiche dell'Università di Zagabria sasa.bjelobaba@fpzg.hr

Con lo scopo di fornire un apprezzamento critico dell'evoluzione della descrizione sincronica dell'italiano standard all'interno della grammaticografia italiana, ci focalizziamo sulla questione del trattamento della categoria delle preposizioni, specificamente sulla classe delle preposizioni proprie nelle grammatiche pubblicate nel secondo Novecento e nei primi due decenni del nuovo millennio. I modelli della definizione e della classificazione delle preposizioni italiane vengono esaminati e valutati criticamente. Secondo i suddetti parametri individuiamo tre filoni principali all'interno della grammaticografia italiana recente: (i) quello "tradizionalista", (ii) quello "generativista" e (iii) "l'approccio di Schwarze". Quest'ultimo viene valutato come sostanzialmente più innovativo e resistente ai dogmi della tradizione grammaticografica italiana perpetuati da decenni, pur con fievoli tentativi dei grammatici italiani di emendare incongruenze.

Parole chiave: grammaticografia, preposizione, definizione, classificazione, significato

\section{Introduzione}

Partiamo dal presupposto che l'affermazione sapiriana sulla natura fallibile di qualsiasi grammatica sia plausibile. ${ }^{1} \mathrm{Di}$ conseguenza, concediamo che le eventuali incongruenze e lacune identificabili in diverse edizioni della grammatica della lingua italiana possano essere interpretate come manifestazioni di questo tratto della grammatica in generale. Ciò nonostante, posto che dal punto di vista dialettico ogni imperfezione suscita l'esigenza di un'indagine finalizzata all'eventuale cambiamento, si può dedurre che ogni nuova descrizione sincronica

1 Vale la pena ricordare che la spesso citata constatazione del linguista e antropologo statunitense - nella fattispecie "All grammars leak." - si riferisce in primo luogo alla grammatica vista come un meccanismo immanente di ogni lingua. L'autore mette in evidenza la proprietà dell'incoerenza di tale meccanismo in quanto in realtà il principio secondo cui le forme analoghe codificherebbero invariabilmente i concetti analoghi, ossia le relazioni analoghe, non di rado risulta violato. Cfr. Sapir (1978: 38). 
della lingua risulterà giustificata, a patto che questa sia mirata ad attenuare le imperfezioni e/o a colmare le lacune che gli autori che intraprendono tali opere hanno individuato nelle descrizioni grammaticali fornite dai loro predecessori. Da ciò si evince che qualunque contributo alla grammaticografia di una determinata lingua andrebbe motivato dall'aspirazione alla progressione evolutiva - tanto a livello descrittivo (per motivi della veracità e dell'attendibilità scientifica), quanto a livello normativo (con lo scopo di raggiungere un sufficiente grado di coerenza con i dati empirici, una condizione favorevole per rendere attuabile una norma della lingua standard in questione).

\section{L'obiettivo della ricerca e il procedimento}

Questa ricerca si prefigge l'obiettivo di stabilire qual è la tendenza principale all'interno della grammaticografia italiana recente, nonché di individuarne eventuali filoni, valutandoli criticamente. Alla luce delle considerazioni sopraesposte, selezioniamo la categoria delle preposizioni come cartina al tornasole dell'eventuale propensione della grammaticografia italiana all'innovazione o, d'altra parte, della tendenza dei grammatici ad attenersi ai canoni dell'autorevole seppur ingombrante tradizione grammaticografica italiana. Una tale selezione è motivata dal fatto che nel corso della storia della linguistica, gli studiosi hanno raramente prestato un'attenzione specifica a questa parte del discorso, soprattutto al suo aspetto semantico. Tuttavia, ciò viene radicalmente cambiato con l'avvento della linguistica cognitiva alla fine del secolo scorso, ovvero una rivolta semantica a livello della linguistica internazionale, l'evento che coincide storicamente con l'inizio di un periodo di notevole fioritura della grammaticografia italiana. ${ }^{2} \mathrm{La}$ nostra analisi è perciò impostata cronologicamente e racchiude un corpus di tredici pubblicazioni uscite tra il 1951 e il 2016: Battaglia/Pernicone ( ${ }^{2} 1977$ [1 ${ }^{\text {a }}$ ed.1951]), Regula/Jernej (1975 [1ª ed. 1965]), Lepschy/Lepschy (1981), Dardano/ Trifone ( ${ }^{2} 1985$ [1 ${ }^{\text {a }}$ ed. 1982]), Rizzi (1988) in Renzi/Salvi/Cardinaletti ( $2001: 7$ [1 ${ }^{\text {a }}$ ed. 1988]), Serianni (Castelvecchi) (1988), Sensini (1997), Andorno (2003), Salvi/ Vanelli (2004), Patota (2006), Trifone/Palermo (2007), Schwarze (2009), e Ferrari/ Zampese (2016). Confrontando in ordine cronologico i modelli della definizione e della classificazione delle preposizioni, ci soffermiamo sulla questione della classe delle preposizioni proprie, un retaggio della tradizione grammaticale italiana. Mettiamo sotto scrutinio l'identità di questa classe esaminando la validità dei criteri secondo cui vengono determinati i membri di questo gruppo. In effetti, le preposizioni assegnate tradizionalmente a questa classe costituiscono il gruppo

2 Si noti la prossimità cronologica tra l'anno 1987 in cui furono pubblicate le due opere fondatrici della linguistica cognitiva (Langacker 1987; Lakoff 1987) e l'anno 1988 che segna la fine del lungo periodo di vacche magre della grammaticografia italiana, l'epoca soprannominata così da Renzi/Salvi/Cardinaletti (2001: 7) per il fatto che quell'anno vide la pubblicazione delle due grandi grammatiche italiane (Serianni 1988; Renzi/Salvi/ Cardinaletti (22001: 7 [1 $1^{\text {a }}$ ed. 1988]) alle quali seguirono le pubblicazioni di numerose altre grammatiche. 
al quale si limita maggiormente l'analisi semantica delle preposizioni all'interno delle grammatiche. ${ }^{3}$

\section{Discussione}

\subsection{Definizioni delle preposizioni nelle grammatiche italiane}

La descrizione sincronica di una categoria grammaticale che pretende di essere completa non può prescindere da una definizione che specifichi i tratti distintivi che i suoi membri hanno in comune, delineando in tal modo i suoi confini. Nelle grammatiche comprese dal corpus è stata notata la prevalenza di criteri sintattici con cui vengono individuati i tratti distintivi rilevanti, nonché la costanza degli autori nell'assegnare la proprietà dell'invariabilità ai membri di questa categoria. Queste osservazioni sono documentate dai dati riportati nella tabella 1 che comprende le grammatiche pubblicate prima dell'anno 1987, che convenzionalmente rimarca l'avvento dell'approccio cognitivo a livello della linguistica internazionale, e nella tabella 2, da cui si può discernere la direzione evolutiva del boom grammaticografico italiano che si verifica a partire dall'anno 1988.

Tabella 1. Le definizioni nelle grammatiche pubblicate prima del 1987

\begin{tabular}{|c|c|c|c|c|}
\hline $\begin{array}{l}\text { Riferimento } \\
\text { bibliografico }\end{array}$ & $\begin{array}{l}\text { Battaglia/Pernicone } \\
\left({ }^{2} 1977\left[1^{\mathrm{a} e d} .1951\right]: 246\right)\end{array}$ & $\begin{array}{l}\text { Regula/Jernej } \\
\text { (1975 [1ª ed. 1965]: 245) }\end{array}$ & $\begin{array}{l}\text { Lepschy/Lepschy } \\
(1981: 95)\end{array}$ & $\begin{array}{l}\text { Dardano/Trifone } \\
\left({ }^{2} 1985 \text { [1 } 1^{\mathrm{a}} \text { ed. 1982]: 263) }\right.\end{array}$ \\
\hline Definizione & $\begin{array}{l}\text { "Le preposizioni } \\
\text { costituiscono il nesso, } \\
\text { potremmo dire il cemento } \\
\text { che rinsalda una parola } \\
\text { all'altra, un elemento della } \\
\text { frase con quello vicino." }\end{array}$ & $\begin{array}{l}\text { "La preposizione è una } \\
\text { particella invariabile del } \\
\text { discorso che, unita a un } \\
\text { termine, forma con esso i } \\
\text { più svariati «tassemi» } \\
\text { (oggetto, attributo, } \\
\text { predicativo, avverbiale, } \\
\text { circostanziale, } \\
\text { giudicativo)." }\end{array}$ & $\begin{array}{l}\text { "Tradizionalmente } \\
\text { vengono considerate } \\
\text { preposizioni in } \\
\text { italiano le seguenti } \\
\text { forme non accentate } \\
\text { (proclitiche: } \\
\text { formano un gruppo } \\
\text { accentuale con il } \\
\text { sintagma seguente): } \\
\text { a, con da, di, fra, in, } \\
\text { per, su, tra." }\end{array}$ & $\begin{array}{l}\text { "Le preposizioni sono } \\
\text { parole invariabili che } \\
\text { servono a collegare e a } \\
\text { raccordare tra loro i } \\
\text { costituenti della } \\
\text { proposizione: } \\
\text { vado a casa di Maria } \\
\text { o a raccordare tra loro due } \\
\text { o più proposizioni: } \\
\text { vado a casa di Maria per } \\
\text { studiare." }\end{array}$ \\
\hline $\begin{array}{l}\text { Proprietà } \\
\text { dell'invariabilità }\end{array}$ & no & sì & no & sì \\
\hline $\begin{array}{l}\text { Criterio } \\
\text { dominante }\end{array}$ & sintattico & sintattico & fonologico & sintattico \\
\hline
\end{tabular}

Ad eccezione della determinazione della categoria proposta da Lepschy/ Lepschy (1981: 95) che non ha pretese di esaustività, il filo conduttore che accomuna altre tre definizioni, indipendentemente dal tipo del metalinguaggio usato, è una netta prevalenza della prospettiva sintattica priva di alcun accenno all'aspetto semantico di questa parte del discorso. Questa tendenza viene ripresa

3 In questo articolo vengono adoperati prevalentemente i risultati di una ricerca più estesa, condotta con lo scopo di contribuire in modo preliminare alla progettazione del nuovo modello dell'analisi semantica delle preposizioni spaziali nell'italiano standard, proposto all'interno dell'omonima tesi del dottorato (Bjelobaba 2018). La tesi è stata realizzata sotto la supervisione della Prof.ssa Ivica Peša Matracki e della Prof.ssa Milena Žic Fuchs. 
in buona parte anche dagli autori del secondo periodo analizzato, come è evidente dalla tabella 2 .

Tabella 2. Le definizioni nelle grammatiche pubblicate dopo il 1987 fino a Schwarze (2009)

\begin{tabular}{|c|c|c|c|c|}
\hline $\begin{array}{l}\text { Riferimento } \\
\text { bibliografico }\end{array}$ & $\begin{array}{l}\text { Serianni (Castelvecchi) } \\
\text { (1988: 279) }\end{array}$ & $\begin{array}{l}\text { Sensini } \\
(1997: 360)\end{array}$ & $\begin{array}{l}\text { Patota } \\
(2006: 60)\end{array}$ & $\begin{array}{l}\text { Trifone/Palermo } \\
\text { (2007: 158) }\end{array}$ \\
\hline Definizione & $\begin{array}{l}\text { "La preposizione è una } \\
\text { parte del discorso } \\
\text { invariabile che serve a } \\
\text { esprimere e determinare i } \\
\text { rapporti sintattici tra le varie } \\
\text { componenti della frase. } \\
\text { Ciascuna preposizione è } \\
\text { dotata di tratti semantici } \\
\text { autonomi, ma nello stesso } \\
\text { tempo è un elemento che ha } \\
\text { funzione relazionale, e } \\
\text { dunque il suo significato si } \\
\text { può cogliere solo in } \\
\text { ragione: a) del tipo di } \\
\text { reggenza che si determina } \\
\text { nell'incontro componente }+ \\
\text { preposizione + componente; } \\
\text { b) dei significati delle } \\
\text { singole parole che si } \\
\text { collegano attraverso la } \\
\text { preposizione." }\end{array}$ & $\begin{array}{l}\text { "La preposizione è quella } \\
\text { parte invariabile del } \\
\text { discorso che si premette a } \\
\text { un elemento della frase } \\
\text { (nome, pronome, verbo } \\
\text { all'infinito, avverbio) per } \\
\text { metterlo in relazione con } \\
\text { un altro elemento della } \\
\text { frase" }\end{array}$ & $\begin{array}{l}\text { "La preposizione è } \\
\text { una parola } \\
\text { invariabile che } \\
\text { precede - a seconda } \\
\text { dei casi - un nome, } \\
\text { un pronome o un } \\
\text { verbo all'infinito } \\
(\ldots) "\end{array}$ & $\begin{array}{l}\text { "Le preposizioni sono } \\
\text { parole invariabili che } \\
\text { servono a mettere in } \\
\text { relazione due elementi } \\
\text { della frase (...). Le } \\
\text { preposizioni svolgono } \\
\text { funzioni distinte nella } \\
\text { frase semplice e in quella } \\
\text { complessa. Nella frase } \\
\text { semplice, servono a } \\
\text { determinare la funzione } \\
\text { logica dell'elemento che } \\
\text { precedono (...) Nella frase } \\
\text { complessa, introducono } \\
\text { varie proposizioni } \\
\text { subordinate implicite e } \\
\text { hanno una funzione } \\
\text { analoga a quella delle } \\
\text { congiunzioni (...). }\end{array}$ \\
\hline $\begin{array}{l}\text { Proprietà } \\
\text { dell'invariabilità }\end{array}$ & sì & sì & sì & sì \\
\hline $\begin{array}{l}\text { Criterio } \\
\text { dominante }\end{array}$ & sintattico & sintattico & sintattico & sintattico \\
\hline
\end{tabular}

Nella tabella 2 non sono incluse le grammatiche appartenenti al filone generativista per il fatto che Rizzi (1988: 521) e Salvi/Vanelli (2004: 173), piuttosto che proporre definizioni esplicite della preposizione, determinano il ruolo sintattico di questa categoria tramite l'analisi della distribuzione potenziale di una preposizione come testa di un sintagma preposizionale. Inoltre, non è inclusa la pubblicazione Andorno (2003: 40) visto che l'autrice si astiene dal proporre una nuova definizione di questa parte del discorso, optando invece di citare quella proposta da Serianni (Castelvecchi) (1988: 279). A nostro giudizio, si tratta della definizione più onnicomprensiva per il fatto che è l'unica a fare riferimento ai membri di questa categoria come portatori di significati autonomi seppur altamente dipendenti dal tipo di reggenza e dai significati delle parole con cui le preposizioni hanno rapporti sintagmatici. Oltre all'evidente prevalenza dell'approccio sintattico, quasi tutti i grammatici attribuiscono il tratto morfologico dell' invariabilità all'intera categoria delle preposizioni italiane. ${ }^{4}$ Sta di fatto che sei membri della categoria delle preposizioni italiane ( $a, d a, d i, i n, s u$ e, in alcuni casi, con) si fondono con le forme dell'articolo determinativo che segue, formando in tal modo i nessi morfologici chiamati preposizioni articolate. Vale a dire, che in questi nessi le forme di e in si trasformano negli allomorfi de- e ne-, mentre, a livello facoltativo, la forma con

4 Gli unici grammatici compresi dal corpus analizzato ad aver evitato l'attribuzione del tratto dell'invariabilità alle preposizioni italiane sono Lepschy/Lepschy (1981: 95-96), Rizzi (1988: 521-545) e Ferrari/Zampese (2016: 99-100). 
cambia in $\mathrm{co}^{-}$, il che rende poco sostenibile la convenzione grammaticale tradizionale secondo cui tutti i membri della categoria vengono proclamati invariabili. Invece, questa incongruenza formale viene emendata da Schwarze (2009: 211) che sostituisce la nozione dell'invariabilità con quella dell'indeclinabilità:

“Le preposizioni sono forme indeclinabili. Alcune di esse però si fondono con l'articolo determinativo, per cui le forme amalgamate hanno la declinazione dell'articolo, (...)" (Schwarze 2009: 211)

Anche se la definizione dell'autore tedesco non differisce considerevolmente da altre definizioni, e nonostante la discutibilità della nozione della declinazione dell'articolo in italiano, questo intervento terminologico può essere interpretato come tentativo di aggiustare un'imperfezione radicata nella tradizione dei grammatici italiani. Vale a dire che la definizione di Schwarze (2009: 211) non si esaurisce con queste osservazioni di indole formale, perché l'autore si sofferma anche sull'aspetto semantico, distinguendo le preposizioni grammaticali da quelle lessicali. ${ }^{5}$ Inoltre, propone due classificazioni separate: quella formale (2009: 211 217) e quella lessicale (2009: 217-221), quest'ultima evidentemente influenzata dai principi dell'approccio cognitivo. Purtroppo, questa scia non viene ripresa dai grammatici successivi. In effetti, l'approccio alle preposizioni italiane di Ferrari/Zampese (2016: 99-100) va associato alla scia tracciata dai predecessori di Schwarze. Oltre all'astensione dall'attribuzione della proprietà dell'invariabilità all'intera categoria, il loro approccio si verifica privo di innovazioni, ma anche di pretese di esaustività.

\subsection{Approccio dei grammatici al gruppo delle preposizioni proprie}

Il gruppo che racchiude le preposizioni italiane prototipiche viene denominato, a seconda degli autori, in diversi modi (v. le tabelle 3 e 4). Si tratta di una categoria relativamente chiusa, per il fatto che la composizione della categoria risulta piuttosto stabile. In realtà, la maggioranza dei grammatici italiani colloca tutte le preposizioni monosillabiche in questo gruppo, pur giustificandone l'appartenenza con un amalgama di argomentazioni entro il quale oscillano, nel senso dell'importanza, i fattori fonologici, morfologici, sintattici, semantici, nonché quello riguardante la frequenza d'uso. In realtà, il nucleo di questa classe è determinato da un unico criterio fonologico latente, ovvero il numero di sillabe.

5 Va notato che Dardano/Trifone (1985: 279), sebbene ai margini della sezione dedicata alle preposizioni, fanno accenno a un'eventuale classificazione delle preposizioni meno tradizionale, affine a quella proposta esplicitamente da Schwarze (2009: 211). 
Tabella 3. Le preposizioni prototipiche italiane nelle grammatiche prima di Andorno (2003)

\begin{tabular}{|c|c|c|c|}
\hline $\begin{array}{l}\text { Riferimento } \\
\text { bibliografico }\end{array}$ & $\begin{array}{c}\text { Denominazione/descrizione } \\
\text { del gruppo }\end{array}$ & Composizione del gruppo & $\begin{array}{l}\text { Argomentazioni adoperate dall'autore } \\
\text { / dagli autori } \\
\text { per giustificare/stabilire la categoria }\end{array}$ \\
\hline $\begin{array}{l}\text { Battaglia/Pernicone } \\
\left({ }^{2} 1977\left[1^{\mathrm{a}} \text { ed.1951]: }\right.\right. \\
246)\end{array}$ & $\begin{array}{l}\text { "(...) quelle }(\ldots) \text { che }(\ldots) \\
\text { sono adoperate con tale } \\
\text { estensione da smarrire il } \\
\text { proprio valore originario, ed } \\
\text { assumere un carattere assai « } \\
\text { generico », che vale a } \\
\text { introdurre le più diverse e } \\
\text { contrastanti relazioni." }\end{array}$ & $\begin{array}{l}a \text { e } d i \text {, in particolare; } \\
\text { e con esse: } d a, i n, p e r, c o n, \\
s u\end{array}$ & $\begin{array}{c}\text { SEMANTICA } \\
\text { valore originario smarrito, carattere } \\
\text { assai generico }\end{array}$ \\
\hline $\begin{array}{l}\text { Regula/Jernej } \\
\text { (1975 [1 } 1^{\mathrm{a}} \text { ed. 1965]: 245) }\end{array}$ & $\begin{array}{l}\text { le preposizioni proprie } \\
\text { (quelle che si uniscono con } \\
\text { l'articolo; preposizioni a } \\
\text { significati semplici) }\end{array}$ & $\begin{array}{l}\text { a (ad), di, da, in, per, con, } \\
\text { su } \\
\text { a significati semplici a) tra, } \\
\text { fra, verso, lungo; b) salvo, } \\
\text { eccetto, tranne, fuorché; } \\
\text { c) durante, mediante, } \\
\text { (concernente, spettante), } \\
\text { nonostante; } d) \text { mercé }\end{array}$ & $\begin{array}{c}\text { MORFOLOGICA } \\
\text { "Si uniscono spesso con l'articolo in una } \\
\text { sola voce (...)" } \\
\text { SEMANTICA } \\
\text { "La significazione di questo gruppo è } \\
\text { molto complessa, introducendo le più } \\
\text { diverse relazioni." }\end{array}$ \\
\hline $\begin{array}{l}\text { Lepschy/Lepschy } \\
(1981: 95-96)\end{array}$ & $\begin{array}{l}\text { "le seguenti forme non } \\
\text { accentate (proclitiche: } \\
\text { formano un gruppo } \\
\text { accentuale con il sintagma } \\
\text { seguente)" }\end{array}$ & $\begin{array}{l}a, \text { con, da, di, fra, in, per, } \\
\text { su, tra }\end{array}$ & $\begin{array}{c}\text { FONOLOGICA } \\
\text { forme non accentate; proclitiche } \\
\text { SINTATTICA } \\
\text { “(...) si possono usare solo soltanto come } \\
\text { preposizioni, a parte } s u \text { che può essere } \\
\text { usato avverbialmente (...)” }\end{array}$ \\
\hline $\begin{array}{l}\text { Dardano/Trifone } \\
\left({ }^{2} 1985\left[1^{\mathrm{a}} \text { ed. } 1982\right]:\right. \\
263-264)\end{array}$ & le preposizioni semplici & $\begin{array}{l}\text { statisticamente più } \\
\text { frequenti: } d i, a \\
\text { seguono per frequenza } \\
\text { d'uso: } d a \text {, in con, su, per, } \\
\text { tra (fra) }\end{array}$ & $\begin{array}{c}\text { FREQUENZA D’USO } \\
\text { “(...) statisticamente più frequenti (...)” } \\
\text { SEMANTICA } \\
\text { “(...) varietà dei significati che esse } \\
\text { esprimono (...)” } \\
\text { MORFOLOGICA } \\
\text { preposizioni articolate (eccetto tra e fra) }\end{array}$ \\
\hline $\begin{array}{l}\text { Rizzi } \\
(1988: 522,525)\end{array}$ & $\begin{array}{l}\text { le preposizioni } \\
\text { monosillabiche }\end{array}$ & $\begin{array}{l}\text { a, con, da, di, fra (tra), in, } \\
\text { per, su }\end{array}$ & $\begin{array}{c}\text { MORFOLOGICA } \\
\text { la formazione di preposizione articolata } \\
\text { SINTATTICA } \\
\text { Non hanno la proprietà dell' estraibilità } \\
\text { del complemento } \\
\text { SEMANTICA } \\
\text { "È problematico indicare un 'nucleo' di } \\
\text { significato costante per ognuna di queste } \\
\text { preposizioni (...)" }\end{array}$ \\
\hline $\begin{array}{l}\text { Serianni (Castelvecchi) } \\
(1988: 283)\end{array}$ & le preposizioni proprie & $\begin{array}{l}\text { di, a, da, in, con, su, per, tra } \\
/ f r a\end{array}$ & $\begin{array}{c}\text { SINTATTICA } \\
\text { “(...) non si impiegano pressoché mai per } \\
\text { altre funzioni sintattiche e grammaticali. } \\
\text { Solo } s u \text {, tra le preposizioni proprie, } \\
\text { conosce un uso anche avverbiale (...)” } \\
\text { MORFOLOGICA } \\
\text { la forma articolata }\end{array}$ \\
\hline $\begin{array}{l}\text { Sensini } \\
(1997: 362)\end{array}$ & le preposizioni proprie & $\begin{array}{l}\text { semplici: di, a, da, in, con, } \\
\text { su, per, tra (fra) } \\
\text { articolate: del, degli, dei, } \\
\text { delle, al, allo ecc. }\end{array}$ & $\begin{array}{c}\text { SINTATTICA } \\
\text { "(...) possono svolgere solo la funzione } \\
\text { di preposizione" } \\
\text { MORFOLOGICA } \\
\text { preposizioni articolate }(d i, \text { a da, in, su; in } \\
\text { qualche caso con, raramente per, mai tra } \\
\text { e fra })\end{array}$ \\
\hline
\end{tabular}

Va notato che solo Regula/Jernej (1975 [1 ${ }^{\text {a }}$ ed. 1965]: 245) affiancano al nucleo di questo gruppo alcune forme polisillabiche, stabilendo in tal modo la sottoclasse di preposizioni proprie a significati semplici. Vi includono le preposizioni monosillabiche sinonime tra e fra, che rimangono disgiunte dal nucleo della classe che comprende il resto delle preposizioni monosillabiche, già annoverate dai predecessori (Battaglia/Pernicone ${ }^{2} 1977$ [1 $1^{\text {a }}$ ed.1951]: 246). A nostro avviso, dal punto di vista formale, la prototipicità delle preposizioni italiane deriva dal potenziale sintattico di una forma preposizionale di svolgere esclusivamente il ruolo preposizionale. Le altre caratteristiche sono secondarie e non determinanti 
per il loro status di prototipo. Al contrario, i grammatici italiani si ostinano a includere in questo gruppo la preposizione avverbiale $s u$ per il mero fatto che essa consiste di una sillaba e poiché si fonde con la forma dell'articolo determinativo che si trova all'inizio del sintagma nominale da essa retto. A questo modello dell'applicazione dei criteri ibridi incapaci di individuare nettamente i membri della categoria, si oppone Andorno (2003: 40) riconoscendo come intrusa la preposizione su e dissociandola dalla classe delle preposizioni proprie.

Tabella 4. Le preposizioni prototipiche italiane nelle grammatiche da Andorno (2003) in poi

\begin{tabular}{|c|c|c|c|}
\hline $\begin{array}{l}\text { Riferimento } \\
\text { bibliografico }\end{array}$ & $\begin{array}{c}\text { Denominazione/descrizione } \\
\text { del gruppo }\end{array}$ & Composizione del gruppo & $\begin{array}{l}\text { Argomentazioni adoperate dall'autore } \\
\text { / dagli autori } \\
\text { per giustificare/stabilire la categoria }\end{array}$ \\
\hline $\begin{array}{l}\text { Andorno } \\
(2003: 39-40)\end{array}$ & le preposizioni proprie & $\begin{array}{l}\text { di, a, da, in, con, per, tra, } \\
\text { fra }\end{array}$ & $\begin{array}{c}\text { SINTATTICA } \\
\text { (...) elementi che svolgono } \\
\text { esclusivamente la funzione di } \\
\text { preposizioni (...)" }\end{array}$ \\
\hline $\begin{array}{l}\text { Salvi/Vanelli } \\
(2004: 173)\end{array}$ & $\begin{array}{l}\text { le preposizioni proprie o } \\
\text { monosillabiche }\end{array}$ & $\begin{array}{l}\text { di, a, da, in, con, su, per, tra } \\
\text { (fra) }\end{array}$ & $\begin{array}{c}\text { FONOLOGICA } \\
\text { forme atone } \\
\text { SINTATTICA } \\
\text { non hanno un uso intransitivo (tranne su) } \\
\text { MORFOLOGICA } \\
\text { preposizioni articolate }\end{array}$ \\
\hline $\begin{array}{l}\text { Patota } \\
(2006: 60)\end{array}$ & $\begin{array}{l}\text { le preposizioni più usate in } \\
\text { italiano }\end{array}$ & $\begin{array}{l}\text { a, con, da, di, fra (tra), in, } \\
\text { per, su }\end{array}$ & $\begin{array}{c}\text { FREQUENZA D’USO } \\
\text { "(...) più usate in italiano (...)" } \\
\text { SEMANTICA } \\
\text { "Esse indicano vari tipi di rapporti fra le } \\
\text { parole che collegano." }\end{array}$ \\
\hline $\begin{array}{l}\text { Trifone/Palermo } \\
\text { (2007: 158) }\end{array}$ & $\begin{array}{l}\text { le preposizioni proprie, o } \\
\text { semplici }\end{array}$ & $\begin{array}{l}\text { di, a, da, in, con, su, per, tra } \\
\text { (o fra) }\end{array}$ & $\begin{array}{c}\text { SINTATTICA } \\
\text { “(...) hanno unicamente funzione di } \\
\text { preposizione (...)" } \\
\text { FONOLOGICA } \\
\text { una sillaba; preposizioni articolate }\end{array}$ \\
\hline $\begin{array}{l}\text { Schwarze } \\
(2009: 211-212)\end{array}$ & le preposizioni semplici & $\begin{array}{l}\text { a, con, da, di, entro, fra, in, } \\
\text { lungo, per, salvo, secondo, } \\
\text { senza, tra, tranne, verso }\end{array}$ & $\begin{array}{c}\text { SINTATTICA } \\
\text { evidente dall'estensione della categoria }\end{array}$ \\
\hline $\begin{array}{l}\text { Ferrari/Zampese } \\
(2016: 99)\end{array}$ & le preposizioni proprie & $\begin{array}{l}\text { di, a, da, in, con, su, per, } \\
\text { traffra }\end{array}$ & $\begin{array}{c}\text { SINTATTICA } \\
\text { “(...) possono fungere esclusivamente da } \\
\text { preposizione (...)" } \\
\text { SEMANTICA } \\
\text { "(...) il loro significato dipende in larga } \\
\text { parte dagli elementi posti in relazione." } \\
\text { MORFOLOGICA } \\
\text { preposizioni articolate } \\
\end{array}$ \\
\hline
\end{tabular}

Come è evidente dalla tabella 4, l'innovazione che Andorno (2003) introduce alla classificazione delle preposizioni italiane non attecchisce nelle opere dei suoi successori. Il primo autore che si riallaccia a questo modello nitido di classificazione formale, appoggiato a un criterio chiaro anziché a un conglomerato eterogeneo di argomentazioni con cui si vela il vero criterio latente - il numero di sillabe, è Schwarze (2009). L'autore tedesco completa la categoria di preposizioni formalmente prototipiche delineata da Andorno (2003) aggiungendo elementi polisillabici il cui comportamento sintattico è altrettanto puramente preposizionale quanto quello delle preposizioni $a, c o n, d a$, di, in, per e tra (fra). 


\section{Conclusione}

In base ai fenomeni osservati, si possono individuare tre direzioni all'interno della grammaticografia italiana nel periodo dal 1951 al 2016.

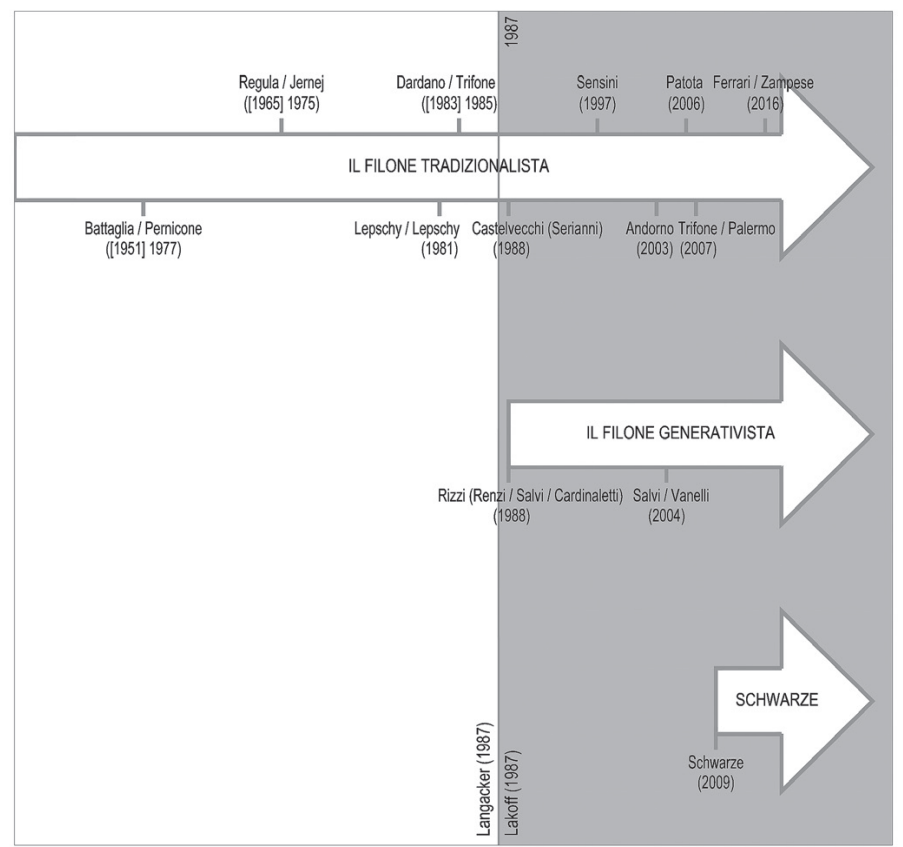

Diagramma 1. Tre direzioni all'interno della grammaticografia italiana dal 1951 al 2016

Relativamente alle grammatiche incluse nel corpus, il filone tradizionalista si rivela indiscutibilmente dominante, sia per il numero delle grammatiche collocate in questo gruppo, sia per l'influsso esercitato sulla classificazione delle preposizioni italiane anche dai grammatici appartenenti al filone generativista, il quale si distingue dalle grammatiche di impronta prevalentemente tradizionale per il suo approccio generale alla descrizione sincronica della categoria delle preposizioni. Prendendo in considerazione il valore dei contributi alla delimitazione coerente della classe delle preposizioni proprie, vanno sottolineati gli interventi innovativi di Regula/ Jernej (1975 [1 $1^{\text {a }}$ ed. 1965]: 245), e in particolare quello della Andorno (2003: 3940). D'altra parte, i confini della categoria delle preposizioni semplici proposta da Schwarze (2009: 211-212) risultano più convincenti per il fatto che l'appartenenza al gruppo è stata determinata tramite l'applicazione coerente di un unico criterio sintattico ben definito. Oltre a ciò, l'approccio di Schwarze si rivela il più innovativo, essendo maggiormente permeato da conoscenze generate da recenti studi semantici a livello internazionale. Si tratta del primo tentativo di fornire una classificazione delle preposizioni italiane basata sui criteri semantici (non solo formali) e di incorporare le nozioni rilevanti scaturite all'interno del quadro generale della linguistica cognitiva nella descrizione semantica delle preposizioni 
italiane. Infine, attribuiamo questo risultato all'autonomia dell'autore tedesco che, emancipato dai modelli tradizionalisti perpetuati dagli autori italiani da decenni, apre la prospettiva di una "ribalta semantica" all'interno della grammaticografia italiana. Nondimeno, un eventuale effetto reale di Schwarze sullo sviluppo della grammaticografia italiana nel suo insieme rimane altamente discutibile a causa della rigidità, del conservatorismo e della propensione dei grammatici italiani ad attenersi in modo dogmatico alla tradizione, nonché per la comprovata renitenza degli autori ad abbracciare le innovazioni positive introdotte dai loro predecessori.

\section{Bibliografia}

Andorno, Cecilia (2003). La grammatica italiana, Milano: Paravia Bruno Mondadori Editori.

Battaglia, Salvatore / Pernicone, Vincenzo ( $\left.{ }^{2} 1977\right)$. Grammatica italiana, la metrica - la stilistica, esercizi e letture lessicali, Torino: Loescher editore [1 $\left.1^{\mathrm{a}} \mathrm{ed} .1951\right]$.

Bjelobaba, Saša (2018). Semantička analiza prostornih prijedloga u standardnom talijanskom jeziku (Tesi di dottorato non pubblicata). Filozofski fakultet Sveučilišta u Zagrebu, Zagreb

Castelvecchi, Alberto (1988). “Capitolo VIII. La preposizione”, in: Serianni, Luca (con la collaborazione di Alberto Castelvecchi). Grammatica italiana. Italiano comune e lingua letteraria. Suoni. Forme. Costrutti, Torino: UTET, pp. 279-303.

Dardano, Maurizio / Trifone, Pietro (1985). La lingua italiana. Morfologia. Sintassi. Fonologia. Formazione delle parole. Lessico. Nozioni di linguistica e sociolinguistica, Bologna: Zanichelli [1 $1^{\text {a }}$ ed. 1982].

Ferrari, Angela / Zampese, Luciano (2016). Grammatica: parole, frasi, testi dell'italiano, Roma: Carocci.

Lakoff, George (1987). Women, Fire and Dangerous Things, What Categories Reveal about the Mind, Chicago - London: The University of Chicago Press.

Langacker, Ronald Wayne (1987). Foundations of Cognitive Grammar, Theoretical Prerequisites, Vol. 1, Stanford: Stanford University Press.

Lepschy, Anna Laura / Lepschy, Giulio (1981). La lingua italiana; Storia, varietà dell'uso, grammatica, Milano: Tascabili Bompiani.

Patota, Giuseppe (2006). Grammatica di riferimento dell'italiano contemporaneo, Novara: Garzanti linguistica.

Regula, Moritz / Jernej, Josip ('21975). Grammatica italiana descrittiva su basi storiche i psicologiche, Bern: Francke Verlag [1 $1^{\text {a }}$ ed. 1965].

Renzi, Lorenzo / Salvi, Giampaolo / Cardinaletti, Anna (22001). Grande grammatica italiana di consultazione, Vol.1, La frase. I sintagmi nominale e preposizionale, Bologna: il Mulino [1 ${ }^{\mathrm{a}}$ ed. 1988].

Rizzi, Luigi (22001). "Il sintagma preposizionale", in: Grande grammatica italiana di consultazione, Vol.1, La frase. I sintagmi nominale e preposizionale, (a cura di Lorenzo Renzi / Giampaolo Salvi / Anna Cardinaletti), Bologna: il Mulino [1 ${ }^{\text {a }}$ ed. 1988], pp. 521-545.

Salvi, Giampaolo / Vanelli Laura (2004). Nuova grammatica italiana, Bologna: il Mulino. 
Sapir, Edward (1978). Language. An Introduction to the Study of Speech, London / Toronto / Sydney / New York: Hart Davis, MacGibbon, Granada Publishing [1 ${ }^{\text {a }}$ ed. 1921].

Schwarze, Christoph (2009). Grammatica della lingua italiana, Roma: Carocci. Sensini, Marcello (1997). La grammatica della lingua italiana, Milano: Mondadori. Serianni, Luca (con la collaborazione di Alberto Castelvecchi) (1988). Grammatica italiana. Italiano comune e lingua letteraria. Suoni. Forme. Costrutti, Torino: UTET Trifone, Pietro / Palermo, Massimo (2007). Grammatica italiana di base, Bologna: Zanichelli.

\section{Talijanska gramatikografija posljednjih desetljeća - u brazdi klasične tradicije ili na raskrižju dekadencije i inovacije?}

U cilju kritičkoga sagledavanja razvoja sinkronijskoga opisa standardnoga talijanskog jezika usredotočujemo se na pitanje pristupa kategoriji prijedloga u djelima novije talijanske gramatikografije. Naime, ova je vrsta riječi tijekom jezikoslovne povijesti rijetko bila u glavnom žarištu pozornosti znanstvenika, a značenjska je analiza članova ove kategorije i na svjetskoj razini bila uvelike zanemarena sve do osamdesetih godina prošloga stoljeća, odnosno pojave kognitivnolingvističkih pristupa. Stoga se upravo pristup gramatičara ovoj kategoriji nameće kao relevantan pokazatelj otvorenosti spram suvremenih jezikoslovnih uvida i tendencija, štoviše kao svojevrsni lakmus papir za utvrđivanje njihove ukalupljenosti u gramatičku tradiciju odnosno autorske inovativnosti. Istraživanje provodimo na korpusu od trinaest gramatika objavljenih u drugoj polovici dvadesetoga stoljeća i u prva dva desetljeća novoga tisućljeća: Battaglia/Pernicone ( ${ }^{2} 1977$ [1 $1^{\text {a }}$ ed.1951]), Regula/Jernej (1975 [1ª ed. 1965]), Lepschy/Lepschy (1981), Dardano/Trifone (21985 [1 $1^{\text {a }}$ ed. 1982]), Rizzi (1988), Serianni (Castelvecchi) (1988), Sensini (1997), Andorno (2003), Salvi/Vanelli (2004), Patota (2006), Trifone/Palermo (2007), Schwarze (2009), te Ferrari/Zampese (2016). Ispitujemo i kritički ocjenjujemo obrasce definiranja kategorije prijedloga u navedenim gramatikama i određivanja skupine pravih prijedloga. Vodeći se ovim kriterijima, utvrđujemo postojanje triju pravaca novije talijanske gramatikografije: (i) „tradicionalističkoga“, (ii) "generativističkoga“ i (iii) „Schwarzeova pristupa“. Potonji ocjenjujemo najinovativnijim i najprožetijim suvremenim lingvističkim uvidima, a ovakav rezultat pripisujemo autonomnosti njemačkoga autora, koji je, oslobođen od čvrstih obrazaca talijanske gramatičke tradicije, otvorio perspektivu semantičkoga obrata u gramatičkoj obradi talijanskih prijedloga. Usprkos tome, stvaran učinak ovakvoga pristupa na talijansku gramatikografiju u cijelosti i dalje ostaje upitan uslijed još uvijek prevladavajuće krutosti, konzervativnosti i dogmatskoga oslanjanja na konvencionalne postavke talijanske gramatičke tradicije, te uočenoga ignoriranja novih znanstvenih spoznaja, ali i pozitivnih noviteta ranije objavljenih gramatika drugih autora.

Ključne riječi: gramatikografija, prijedlog, definicija, klasifikacija, značenje 